\title{
Point-of-Care Testing vs. Laboratory Testing during High Patient Volume Situations
}

\author{
Jonathan McCoy ${ }^{*}{ }^{\circledR}$, Robert Eisenstein'1, Channing Hui' ${ }^{2}$, Greg Corcoran³, Chris Kilker1, \\ Pamela Ohman-Strickland4, Mark Merlin ${ }^{5}$, Clifton Lacy ${ }^{6}$
}

\author{
${ }^{1}$ Rutgers-Robert Wood Johnson Medical School, Department of Emergency Medicine (RWJMS), New Brunswick, NJ, USA \\ ${ }^{2}$ Alpert Medical School of Brown University, Department of Medicine, Division of Pulmonary, Critical Care, and Sleep Medicine, \\ Providence, RI, USA \\ ${ }^{3}$ Capital Health Emergency Department, Trenton, NJ, USA \\ ${ }^{4}$ Rutgers-School of Public Health (SPH), Piscataway, NJ, USA \\ ${ }^{5}$ Newark Beth Israel Medical Center, Department of Emergency Medicine, Newark, NJ, USA \\ ${ }^{6}$ Rutgers-Robert Wood Johnson Medical School, Rutgers School of Communication and Information, New Brunswick, NJ, USA \\ Email: *mccoyj1@rwjms.rutgers.edu
}

How to cite this paper: McCoy, J., Eisenstein, R., Hui, C., Corcoran, G., Kilker, C., Ohman-Strickland, P., Merlin, M. and Lacy, C. (2019) Point-of-Care Testing vs. Laboratory Testing during High Patient Volume Situations. Open Journal of Emergency Medicine, 7, 49-56. https://doi.org/10.4236/ojem.2019.74006

Received: December 3, 2019

Accepted: December 27, 2019

Published: December 30, 2019

Copyright $\odot 2019$ by author(s) and Scientific Research Publishing Inc. This work is licensed under the Creative Commons Attribution International License (CC BY 4.0).

http://creativecommons.org/licenses/by/4.0/ (c) (i) Open Access

\begin{abstract}
Introduction: Our aim was to determine what patient volume, if any, in-laboratory testing provides results faster than Point-of-Care-Testing (POCT). Methods: To evaluate POCT effectiveness during high volume situations, POCT was compared to in-laboratory testing during busy periods with large numbers of patients. Our setting was an urban level 1 trauma center with an academic emergency medicine department (ED) and annual patient volume of 70,000. Patients seen requiring laboratory testing during peak volume between 11 a.m. and 7 p.m. were enrolled over a five-week period. One tube of blood was sent to the laboratory and the other tube was run in the ED using POCT. Turnaround time was recorded as time from when the tube was received to when the result was available. We also completed a time-motion study to assess the number of POCT machines that would be needed to process the entire average hourly hospital laboratory volume. Results: We collected 539 hematology and chemistry specimens. The POCT group was significantly faster than in-laboratory testing, with mean POCT [complete blood count ( $\mathrm{CBC}$ ) and chemistry] 3.5 minutes compared to in-laboratory $\mathrm{CBC}$ test time of 30.9 minutes and chemistry test time of 55 minutes. As the volume of samples peaked, there was a slight but insignificant decrease in POCT turnaround time. If POCT was used to process the entire average hospital laboratory volume which approached 54 samples an hour, 3 POCT machines would be necessary to maintain turnaround times. Conclusion: Even during ED high volume situations, POCT provided results significantly faster than in-laboratory testing.
\end{abstract}




\section{Keywords}

Point-of-Care Testing, Emergency Department, Disaster Medicine

\section{Introduction}

Our nation's Emergency Departments (EDs) are in a state of crisis [1]. Crowding and long wait times have become commonplace. This has led to a demand for improved process efficiency and decreased turnaround times (TAT). Point-ofCare-Testing (POCT) has the potential to help accomplish this goal, since the accuracy of these devices has been shown to be equivalent to in-laboratory testing [2]. Decreased ED TAT in selected patient populations may help alleviate ED crowding [3] [4].

Several studies support the use of POCT for cardiac markers in patients with chest pain [5]-[9]. However, POCT has not been specifically compared to in-laboratory testing in high volume busy or mass casualty situations [10]. For example, even if the benefit can be shown in individual patients, what happens when there is a large volume of patients that require testing at the same time? Will the tenth POCT sample have the same logistic benefit as the first or will the laboratory's capacity to run several samples simultaneously result in a quicker result? How many POCT machines would be required to match the laboratory's ability to accommodate a high volume situation?

Given the need to develop strategies to adapt to high volumes, the results of this study could potentially identify a way to improve efficiency under these circumstances. Several studies have demonstrated that POCT decreases length of stay in the ED, providing evidence that POCT can alleviate ED crowding [11] [12] [13]. However, we acknowledge that having results faster does not always translate to short ED stays.

\section{Objective}

To evaluate the effectiveness of the POCT during high volume situations, POCT was compared to in-laboratory testing during busy periods with large numbers of patients.

\section{Methods}

\subsection{Study Design, Setting, and Selection of Participants}

This study was completed at an urban level 1 trauma center with an academic emergency medicine department with a residency and annual patient volume of 70,000 . Blood samples requiring laboratory testing during the afternoon peak volume between 11 a.m. and 7 p.m. were included over a five-week period beginning January, 2012. These hours were chosen since review of Emergency Department Information manager (EDIM) demonstrated the highest volume of patients within these hours. 


\subsection{Methods, Measurements, and Outcomes Measured}

Blood samples were drawn in the usual manner. One tube of blood was tested in the ED using POCT with the GEM Premier 4000 (Bedford, MA) and the others were sent to the laboratory for testing. The GEM Premier 4000 has a limited laboratory panel, including $\mathrm{pH}, \mathrm{pCO} 2, \mathrm{pO} 2, \mathrm{Na}+, \mathrm{K}+, \mathrm{Ca}++, \mathrm{Cl}-$, glucose, lactate, hematocrit, total hemoglobin, oxyhemoglobin, carboxyhemoglobin, methemoglobin, deoxyhemoglobin, and total bilirubin. TAT was measured as time from when the tube was received to when the result was available, and each test was captured and compared. POCT was completed in the ED by a laboratory specialist stationed in the ED to complete the POCT and ensure all the appropriate quality control maintenance was done. All patients were treated in the usual manner of care. The study received a non-human subject research exemption from the Institutional Review Board.

A supplemental time-motion study was completed to detail the steps involved in lab processing, characterize the manual labor involved in laboratory tests, and estimate the number of POCT machines that would be necessary to handle the complete hospital volume in an hour.

\subsection{Analysis}

We compared POCT times from receipt to results for complete blood count (CBC) and chemistries with in-laboratory $\mathrm{CBC}$ and chemistry times. If the in-laboratory result time preceded the documented time the sample was received, the sample time was "negative". It was assumed that the time of receipt was input incorrectly, and the observation was excluded. This was to account for samples dropped off to the POCT station prior to nursing completing collection status in the computer and sending samples to the labs. This would overestimate POCT times. If either the POCT or in-laboratory sample was not received, the observation was excluded. Missing values were not imputed. Descriptive statistics were used to summarize the sample distribution. For both POCT and in-laboratory testing, the numbers of TAT greater than 60, 90 and 120 minutes were calculated. Rank sum tests were used to assess significance of differences in processing time between in-laboratory and POCT.

We also performed a sensitivity analysis to determine whether times for in-laboratory CBC and chemistry labs were longer (relative to POCT) during high volume ED activity. We used scatter plots to examine the association between differences between in-laboratory CBC and chemistry and POCT processing times as a function of number of labs processed in an hour. Weighted linear regression analyses formally examined these associations, where weights were set equal to the number of labs samples received. Observations were grouped into hours of the day the labs were received and mean processing times calculated for each hour compared with the number of samples received. The analyses were repeated using day rather than hour as the unit of accumulation. 


\section{Results}

Over a five-week period, 569 blood test samples were collected during times of peak ED volume. Of 569 observations, 30 (5.3\%) had a negative number or a missing sample pair and were excluded. All analyses were conducted on the remaining 539 observations.

The POCT group was significantly faster than in-laboratory testing, with a mean POCT turnaround time (TAT) of 3.5 minutes, compared with in-laboratory $\mathrm{CBC}$ test time of 30.9 minutes and blood chemistry test time of 55 minutes (Table 1). Of note, while mean times were less than 60 minutes, in-laboratory testing frequently took longer than 60 minutes as described in (Table 2). Our sensitivity analysis found that as the number of samples increased and reached a peak, there was a slight decrease in POCT TAT, but the difference was insignificant. There was no time when in-laboratory testing was faster than POCT.

Since the peak volume of samples per hour did not sufficiently stress either method, we performed a time-motion study to determine the point at which POCT becomes less efficient than in-laboratory testing. The maximum number of samples per hour on a single POCT machine was 17 samples at 3.5 minutes per sample. There were several variables that determined the maximum number of in-laboratory samples per hour. The time-motion study assessed all the steps and time involved in getting specimens from blood draw in ED to result in the laboratory. We found that specimens the laboratory received from the ED were not run separately from those received from the rest of the hospital. Therefore, although we only assessed the TAT for ED specimens, the laboratory was processing many more specimens during the same hour. So, while our highest volume was 8 samples from the ED in an hour, the laboratory received an average of 54 specimens per hour (Figure 1).

If we were to assume all 54 samples arrived at once, similar to a theoretical surge or mass casualty situation, it would take 3 hours to process the 54 specimens using only POCT even if single sample duration remained only $3.5 \mathrm{mi}-$ nutes. Alternatively, 3 POCT machines would be necessary to process all samples within an hour. POCT has the potential to be associated with delay during surge or batch testing situations.

Table 1. Mean and standard deviation of turnaround times (TAT) and distribution of processing turnaround times within 25th, 50th, or 75th percentiles for point-of-care testing (POCT) compared to in-laboratory complete blood count (CBC) and chemistry (Chem) testing. $\mathrm{N}=539$ observations. POCT processing times were significantly less than either in-lab CBC or Chemistry processing times, both $\mathrm{p}<0.0001$ using Sign Rank test).

\begin{tabular}{cccccccc}
\hline Method & $\begin{array}{c}\text { Turnaround } \\
\text { Time Mean } \\
\text { (Minutes) }\end{array}$ & Std Dev & $\begin{array}{c}\text { Distribution: } \\
\text { Minimum }\end{array}$ & $25 \%$ & $50 \%$ & $75 \%$ & Maximum \\
\hline POCT & 3.5 & 4.6 & 1 & 2 & 3 & 3 & 63 \\
CBC In-Lab & 30.9 & 25.5 & 2 & 15 & 24 & 39 & 183 \\
Chem In-Lab & 55.2 & 34.8 & 6 & 35 & 46 & 64 & 424 \\
\hline
\end{tabular}


Table 2. Percent of times when testing modality took longer than 60,90 , or 120 minutes to complete; machine run and manual labor times from detailed time motion study machines that would be necessary to handle the complete hospital volume in an hour.

\begin{tabular}{ccccccc}
\hline Method & Minutes & $\begin{array}{c}\text { \# TAT> }>\text { TAT> } \\
\text { Minutes }\end{array}$ & $\begin{array}{c}\text { Minutes } \\
\text { (SD) Minutes }\end{array}$ & $\begin{array}{c}\text { Median Run } \\
\text { Time Minutes }\end{array}$ & $\begin{array}{c}\text { Mean Manual } \\
\text { Time Minutes }\end{array}$ \\
\hline $\begin{array}{c}\text { POCT } \\
\text { [eN 539] }\end{array}$ & 60 & 2 & 0.4 & 1 & $<1$ & 2.5 \\
& 120 & 0 & 0 & & & \\
& 60 & 51 & 9.7 & $21(23)$ & 10 & 2 \\
CBC In-Lab & 90 & 20 & 3.8 & & & \\
[eN 524] & 120 & 8 & 1.5 & & & \\
& 60 & 153 & 28.5 & $52(20)$ & 45 & \\
Chem & 90 & 54 & 10.1 & & & \\
In-Lab & 120 & 25 & 4.7 & & & \\
[eN 537] & 120 & & & \\
\hline
\end{tabular}

a. Shorter $\mathrm{CBC}$ run times compared to the prior observations reflect changes in laboratory procedures; $\mathrm{b}$. turnaround time (TAT); c. standard deviation (SD); d. eligible $\mathrm{N}(\mathrm{eN})$ refers to 60, 90, 120 minute distributions.

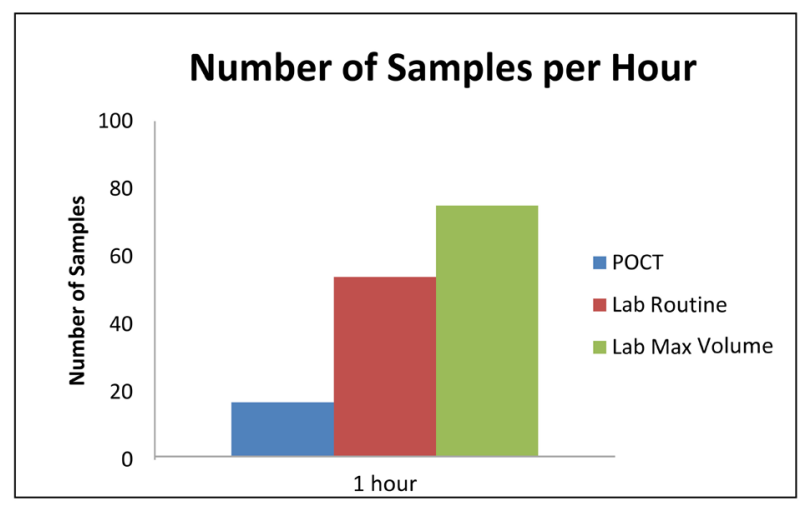

Figure 1. Number of samples processed per hour. The laboratory receives 75 samples per hour during peak times of 5 a.m. to 9 a.m. when all inpatient samples are run and turnaround times are extended. During routine times, the laboratory processes about 54 specimens an hour. If point of care testing (POCT) were to handle the same volume at its maximum rate of 17 samples per hour, POCT could handle the total hospital morning surge volume with 3 systems, with turnaround times measured in minutes rather than tens of minutes.

\section{Discussion}

We found that during routine ED high volume situations, POCT provided results significantly faster than in-laboratory testing. We also found that if POCT were used for total hospital laboratory testing volumes approaching 54 samples an hour, 3 POCT machines would be necessary to maintain rapid turnaround times for most specimens. While POCT holds promise as a solution for fast specimen processing during high volume situations, some caution is advised because most institutions will not typically have the number of POCT systems necessary to maintain fast processing for all specimens within a short period. Fur- 
ther, if samples arrived in a significant batch such as from a mass casualty incident rather than a more evenly distributed daily census, scalability of POCT may be further stressed. However, these concerns remain theoretical given the moderately limited volumes of specimens available in our study.

The time-motion study found that in most instances, the observed manual labor was not very different between POCT or in-laboratory testing. However, in-laboratory TAT was hampered by the many other hospital samples being run simultaneously, with placement of the just arrived sample in a relatively long automated que tray on its way to the laboratory testing machine. This step allowed the laboratory technician to complete other tasks, increasing their efficiency but potentially accounting for the wide variability in laboratory median run times.

Planning for mass casualty incidents perhaps needs to include discussions with the laboratory to limit testing to specimens from the ED and ICUs to temporarily improve system efficiency. If POCT will be the solution to meet high volume demands, the number of systems that may be required in a mass casualty bolus situation should be considered. Moreover, unlike during this trial, in many institutions with POCT, nursing staff perform the test, which may impact their ability to perform other tasks during mass-casualty situations. Nevertheless, in our study, POCT significantly outperformed in-laboratory testing during routine high patient volume situations, affirming its diagnostic efficiency.

We acknowledge a number of limitations. During our busiest high volume times, neither laboratory testing nor POCT system was sufficiently stressed to simulate a mass casualty incident. We attempted to account for this with an additional time motion study and sensitivity analysis. Due to lab concerns for maintenance of quality control, laboratory personnel performed the POCT within the ED, which may not be generalizable to all hospitals.

Additionally, the nursing workflow mechanism for documenting time of collection in our electronic medical record may have affected the actual collection time of some specimens as they may have documented collection well after specimens were already in the laboratory, creating a negative number and causing their data to be excluded from analysis. Rarely, some specimens were sent to the laboratory without a matching POCT specimen and their time data was excluded from analysis.

We anticipated a learning curve in the beginning of the study, with the expectation of increasing samples collected progressively over a five-week period. However, we found the number of samples collected each week decreased, possibly revealing a selection bias. For example, if a patient appeared pale the nurse may favor CBC POCT to obtain faster results to expedite care. In addition, this study excluded weekends and samples from the pediatric emergency department. The blood samples were collected based on our busiest hours, calculated from our ED electronic medical record system. We did not adjust for the day of the week, month, or season, which may have provided a higher patient volume. 


\section{Conclusion}

Point-Of-Care Testing was significantly faster in providing results compared to in-laboratory testing during routine high patient volume situations. However, during a true mass casualty incident requiring simultaneous blood specimen testing, multiple POCT machines may be necessary. Future research on POCT should take into account the effects of workload on staff who will actually perform the test and how that may affect their availability of other duties.

\section{Acknowledgements}

1) This project was sponsored by the University Center for Disaster Preparedness and Emergency Response. This study received funding from the US Department of Defense Grant No. W9132T-10-1-0001. The views, opinions, positions, conclusions, or strategies in this work are those of the authors and do not necessarily reflect those of the Department of Defense or any agency of the U.S. Government and no official endorsement should be inferred.

2) Jonathan McCoy received funding from Boehring-Ingelheim for REVERSE AD site-related costs which do not relate to this work.

\section{Conflicts of Interest}

The authors declare no conflicts of interest regarding the publication of this paper.

\section{References}

[1] Institute of Medicine BoHCS, Committee on the Future of Emergency Care in the United States Health System (2007) Hospital-Based Emergency Care: At the Breaking Point. National Academies Press, Washington.

[2] Jacobs, E., Vadasdi, E., Sarkozi, L. and Colman, N. (1993) Analytical Evaluation of i-STAT Portable Clinical Analyzer and Use by Nonlaboratory Health-Care Professionals. Clinical Chemistry, 39, 1069-1074.

[3] Lee-Lewandrowski, E., Corboy, D., Lewandrowski, K., Sinclair, J., McDermot, S. and Benzer, T.I. (2003) Implementation of a Point-of-Care Satellite Laboratory in the Emergency Department of an Academic Medical Center: Impact on Test Turnaround Time and Patient Emergency Department Length of Stay. Archives of Pathology \& Laboratory Medicine, 127, 456-460.

[4] Lee-Lewandrowski, E., Nichols, J., Van Cott, E., et al. (2009) Implementation of a Rapid Whole Blood D-Dimer Test in the Emergency Department of an Urban Academic Medical Center: Impact on ED Length of Stay and Ancillary Test Utilization. American Journal of Clinical Pathology, 132, 326-331. https://doi.org/10.1309/AJCP6US3ILGEAREE

[5] Renaud, B., Maison, P., Ngako, A., et al. (2008) Impact of Point-of-Care Testing in the Emergency Department Evaluation and Treatment of Patients with Suspected Acute Coronary Syndromes. Academic Emergency Medicine: Official Journal of the Society for Academic Emergency Medicine, 15, 216-224. https://doi.org/10.1111/j.1553-2712.2008.00069.x

[6] Ryan, R.J., Lindsell, C.J., Hollander, J.E., et al. (2009) A Multicenter Randomized Controlled Trial Comparing Central Laboratory and Point-of-Care Cardiac Marker 
Testing Strategies: The Disposition Impacted by Serial Point of Care Markers in Acute Coronary Syndromes (DISPO-ACS) Trial. Annals of Emergency Medicine, 53, 321-328. https://doi.org/10.1016/j.annemergmed.2008.06.464

[7] Kemper, D.W., Semjonow, V., de Theije, F., et al. (2017) Analytical Evaluation of a New Point of Care System for Measuring Cardiac Troponin I. Clinical Biochemistry, 50, 174-180. https://doi.org/10.1016/j.clinbiochem.2016.11.011

[8] Bertsch, T., Chapelle, J.P., Dempfle, C.E., Giannitsis, E., Schwabs, M. and Zerback, R. (2010) Multicentre Analytical Evaluation of a New Point-of-Care System for the Determination of Cardiac and Thromboembolic Markers. Clinical Laboratory, 56, 37-49.

[9] Muller-Bardorff, M., Sylven, C., Rasmanis, G., et al. (2000) Evaluation of a Point-of-Care System for Quantitative Determination of Troponin T and Myoglobin. Clinical Chemistry and Laboratory Medicine, 38, 567-574. https://doi.org/10.1515/CCLM.2000.083

[10] Morrow, D.A., Cannon, C.P., Jesse, R.L., et al. (2007) National Academy of Clinical Biochemistry Laboratory Medicine Practice Guidelines: Clinical Characteristics and Utilization of Biochemical Markers in Acute Coronary Syndromes. Circulation, 115, e356-e375. https://doi.org/10.1161/CIRCULATIONAHA.107.182882

[11] Jang, J.Y., Shin, S.D., Lee, E.J., Park, C.B., Song, K.J. and Singer, A.J. (2013) Use of a Comprehensive Metabolic Panel Point-of-Care Test to Reduce Length of Stay in the Emergency Department: A Randomized Controlled Trial. Annals of Emergency Medicine, 61, 145-151. https://doi.org/10.1016/j.annemergmed.2012.07.021

[12] Murray, R.P., Leroux, M., Sabga, E., Palatnick, W. and Ludwig, L. (1999) Effect of Point of Care Testing on Length of Stay in an Adult Emergency Department. The Journal of Emergency Medicine, 17, 811-814. https://doi.org/10.1016/S0736-4679(99)00107-9

[13] Hsiao, A.L., Santucci, K.A., Dziura, J. and Baker, M.D. (2007) A Randomized Trial to Assess the Efficacy of Point-of-Care Testing in Decreasing Length of Stay in a Pediatric Emergency Department. Pediatric Emergency Care, 23, 457-462. https://doi.org/10.1097/01.pec.0000280506.44924.de 